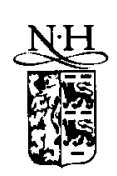

\title{
On solving intransitivities in repeated pairwise choices
}

\author{
Arne Maas, Thom Bezembinder, Peter Wakker* \\ University of Nijmegen (NICI), P.O. Box 9104, Nijmegen, Netherlands \\ Received February 1993 \\ Revised April 1994
}

\begin{abstract}
An operational method is presented for deriving a linear ranking of alternatives from repeated paired comparisons of the alternatives. Intransitivities in the observed preferences are cleared away by the introduction of decision errors of varying importance. An observed preference between two alternatives that causes an intransitivity in the course of the procedure will be reversed if it is of lesser importance. The method is applicable in case one wants to take account of intensities of preference and assume these to be monotone with the probability that an observed choice coincides with a fixed underlying true choice.
\end{abstract}

Keywords: Intransitivities; Paired comparisons; Linear rankings

\section{Purpose and motivation}

Transitivity is a fundamental, if not the most fundamental, notion in decision theory. It is almost universally assumed in disciplines of decision theory and generally accepted as a principle of rationality. Yet, it is often violated in actual choice, particularly in pairwise choices. A first task for decision science is thus the resolution of intransitivities. This is the subject of the present paper. We shall present an algorithm for solving intransitivities in repeated choices.

In many practical decision problems a set of options is given at the outset and it is required that one single option finally emerges from the decision process as 'the best'. Here, the decision process may be of various kinds but we only consider pairwise choices. As, in general, any option may be embedded in an (intransitive) cycle of pairwise preferences, the requirement of one single option emerging as

\footnotetext{
* Corresponding author.
} 
'the best' asks for a decision process in which such cycles are broken and transformed into linear orders.

The method we present was developed in the framework of a medical decision analysis in which treatments for patients of laryngeal cancer (Maas and Stalpers, 1992) had to be determined. To be maximally useful for practical applications, we based our method on two intuitive assumptions:

(i) More intense preferences are more trustworthy.

(ii) Preferences that are invariant over replications are to be preserved.

These assumptions serve to get our method to be transparent, tractable in its details, and mathematically simple.

\section{Introduction}

In the method of paired comparisons, pairs from a set $Z=\{x, y, z, \ldots\}$ of $n \geqslant 3$ objects are presented to one judge. The method is very popular in the social sciences and is used primarily when the objects to be compared cannot be measured in a physically meaningful way. In practical applications ties are often not permitted, that is, each judgment consists of a choice or strict preference (in some sense) for one of the two compared objects. Let us consider the simple case of three objects $x, y$, and $z$ to be judged once by one person, so that there are three pairs to be compared: $\{x, y\},\{y, z\}$ and $\{x, z\}$. Let $>$ denote a binary preference relation on $Z$, where $x>y$ means that $x$ is strictly preferred to $y$, i.e. $x$ (and not $y$ ) is chosen from $\{x, y\}$. As each comparison has two possible outcomes, this yields eight possible experimental results. In six of these results one object is chosen twice, a second object is chosen once, and the third object is not chosen at all. In the other two results each object is chosen once, that is,

$$
\text { either }[x>y, y>z, z>x] \text { or }[y>x, x>z, z>y]
$$

has been observed. Such results are called circular triads (Kendall and Smith, 1940). Obtaining a linear order from such results is a problem that occurs in several fields.

A circular triad may be regarded as an inconsistency of the judge, and several explanations for the occurrence of a circularity can be given (e.g. Tversky, 1969; David, 1988). It should be noted that circularities are not restricted to the case of three objects: $m$ objects may give rise to a circular $m$-ad; we refer to any circular $m$-ad as an intransitivity $(m \geqslant 3)$. However, while intransitivities may occur, researchers are often interested in establishing a transitive order. This may be the case if a prespecified model (e.g. unfolding, conjoint measurement) or a prescriptive application (e.g. in decision analysis) requires transitive data. One widely known procedure (Slater, 1961) consists of finding a linear order on the basis of the observations such that the number of preference reversals is as small 
as possible; this number is known as Slater's $i$. Given intransitive data, all linear orders obtained from the data by exactly $i$ preference reversals are called nearest adjoining orders. Algorithms for finding all nearest adjoining orders can be found in Remage and Thompson (1966) and Phillips (1969); an efficient algorithm for finding $i$ and one nearest adjoining order for $n<10$ objects is given by Bezembinder (1981). See also Goddard (1983), Ali et al. (1986), and Barthélemy (1990).

Slater's procedure has been formulated for the case where one judgment is obtained for each of the $\left(\begin{array}{l}n \\ 2\end{array}\right)$ unordered pairs $\{x, y\}, x \neq y$, that may be formed from the set $Z$ of alternatives under scrutiny. Moon (1968) and, more recently, Delver et al. (1991), mention some other ranking methods for that case. However, in Moon's (1968, p. 42) judgment, none of the methods he considers is 'entirely satisfactory' while none of the methods considered by Delver et al. satisfies all of the properties they deem desirable for these methods.

We present a method that reckons with the extent to which a judge is stable in his/her preferences over repeated trials; this stability will henceforth be interpreted as a measure of the intensity of preference. A natural starting point under replicated choices is the majority preference relation: $x$ is majority preferred to $y$ if $x$ has been chosen from $\{x, y\}$ in the majority of the presentations of $\{x, y\}$. Given an odd number of replications, preferences are thus dichotomized: $x$ is strictly preferred to $y$, or $y$ to $x$. Problems arise if the resulting majority preference relation turns out to be intransitive. Then, in applications, often an ad hoc nearest adjoining order is taken with respect to the majority preference relation. Yet, the dichotomization has led to a loss of information. Our method reckons with all the information of replicated choices. In this respect our method contrasts with dichotomization and all procedures such as Slater's and the ones mentioned above in Moon (1968) and Delver et al. (1991), where all observed choices are implicitly assumed to be of equal importance; see Example 6.2 below. There also exists a large literature on the scaling of a set of objects from a set of pairwise relative choice frequencies (here interpreted as intensities of preferences) that may or may not violate transitivity of majority choice. Often a goal function is chosen that should be maximized. For a survey of related theories, and many sophisticated procedures, see Hubert (1976).

This paper proposes a new method for solving intransitivities. Our method aims at being tractable and natural in practice, and will be tested as such in a computer simulation. Simplicity and appeal, rather than mathematical sophistication, are our primary aims. The method will not consist of maximizing a function. In contrast to Slater (1961), and most other methods such as the ones in Moon (1968) and Delver et al. (1991), our method can be applied to incomplete digraphs representing, for example, the ordinal information embedded in a conditional proximity matrix. We describe our method for the general case of incomplete digraphs, and given some examples and applications, such as to 
Guttman's scalogram model, and social choice theory. Incidentally, for the latter application a feature of our method is that the unanimity principle is satisfied.

We consider stable preferences, i.e. preferences that remain the same throughout all replications, as particularly important. They contain all preferences of high reliability and intensity. Hence they will not be confounded with unstable preferences, which evidently cannot be highly reliable. Stable preferences can be confounded with unstable ones if a nearest adjoining order with respect to majority preferences is chosen, or if a function is maximized. Cycles derived from stable preferences are more likely to represent genuine cycles than those derived from unstable preferences. Imposing a transitive order on a cycle of stable preferences may very well be inappropriate. Hence, in that case our method stops, and reconsideration of the data is recommended. Then, in particular, the data may be reconsidered in the light of decision models that allow genuine intransitivity such as Tversky (1969), Loomes and Sugden (1982), Bell (1982), and Fishburn and LaValle (1987).

Section 5 shows that our method requires polynomial computation time. This reflects the simplicity and tractability mentioned above. About Slater's procedure, David (1988, p. 24) wrote that ". . . the calculation of $i$ (and a nearest adjoining order) continues to present a formidable challenge except when [the number of objects] is small". The methods presented in Remage and Thompson (1966) and Phillips (1969) require exponential computation time. Of course, tractability is especially important for dealing with many objects, and for the programming of the method. It does not allow for indifferences; inter alia, Singh and Thompson (1968) deal with indifferences. Our method preserves completeness, i.e. if the data satisfy completeness, then so does the solution. The method can be applied to incomplete preferences, and usually gives a unique transitive order.

After the preliminaries in Section 3, the method is explained in detail in Section 4. Section 5 shows that the computation time of the method is polynomial. Section 6 gives examples and Section 7 a Monte Carlo study. Section 8 contains the discussion.

\section{Preliminaries}

Let $Z=\{x, y, z, \ldots\}$ be a nonempty set of $n$ objects with $x, y, z$ possibly subscripted. A binary relation $R$ on $Z$ is

(i) asymmetric if $x R y \Rightarrow$ not $y R x$, for all $x, y \in Z$;

(ii) transitive if $x R y, y R z \Rightarrow x R z$, for all $x, y, z \in Z$;

(iii) complete if $x R y$ or $y R x$, for all $x \neq y \in Z$;

(iv) acyclic if, for all $m \geqslant 1,\left[x_{1} R x_{2}, x_{2} R x_{3}, \ldots, x_{m-1} R x_{m}\right] \Rightarrow\left[\operatorname{not} x_{m} R x_{1}\right]$.

$\mathrm{R}$ is a linear order if it satisfies (i), (ii) and (iii), a tournament if it satisfies (i) and (iii) and a partial (or transitive) order if it satisfies (i) and (ii). Obviously, a 
transitive tournament is a linear order. The transitive closure of $R$, denoted by $R^{t}$, is defined by $x R^{\mathrm{t}} y$ if $x R y$ or $x R z_{1} R \ldots R z_{m} R y$ for some $z_{1}, \ldots, z_{m} \in Z$. We let $Z \& Z$ denote the set of the unordered pairs $\{x, y\}, x, y \in Z, x \neq y$, that we plan to present to a subject in a particular experiment. Note that $Z \& Z$ may, but need not, equal the set of all unordered pairs that may be formed from $Z$. Note also that $R$ is transitive if it satisfies (i), (iii), and (iv).

Let $\rightarrow$ be an asymmetric binary relation on $Z . D=\langle Z, \rightarrow\rangle$ is a directed graph, or digraph for short, with objects called nodes, and a relation between nodes is called an arc. We will frequently refer to an arc $x y$ meaning an arc from $x$ to $y$. A walk in a digraph is an alternating sequence of nodes and arcs, where the nodes before and after an arc are its beginning and endnodes, respectively. A path is a walk in which all nodes are distinct. A cycle is a walk with at least three nodes, where all nodes are distinct except the first and the last. A cycle containing $m$ nodes is called an $m$-cycle.

The indegree id $(x)$ of a node $x$ is the number of incoming arcs $y x$, the outdegree $\operatorname{od}(x)$ is the number of outgoing arcs $x y$. Obviously, the sum of in- and outdegree of a node never exceeds $n-1$.

A tournament $T$ of $n$ nodes contains $N=\left(\begin{array}{l}n \\ 2\end{array}\right)$ arcs, and may be used to represent all $N$ pairwise preferences within a set of $n$ objects. We call $s=$ $(\operatorname{od}(1), \ldots, \operatorname{od}(n))$ with $\operatorname{od}(1) \geqslant \cdots \geqslant \operatorname{od}(n)$ the score vector of $T$, where od $(l)$ is the outdegree of node $l$ for $l=1, \ldots, n$. A tournament $T$ is transitive if and only if $s=(n-1, n-2, \ldots, 1,0)$; see Moon (1968, Theorem 9).

An observed choice between two objects, made by a judge, can be called a manifest choice or manifest preference; usually we drop manifest, and simply write 'choice' or 'preference'. Hence, from now on, 'choice' and 'preference' without any specification will refer to manifest choice and manifest preference. We assume that for each $\{x, y\} \in Z \& Z$ there exists a fixed true or latent choice, denoted by $t(x, y)$, and that a manifest choice in $\{x, y\}$, denoted by $m(x, y)$, reflects $t(x, y)$ but may be distorted by error. A probabilistic assumption could be that $m(x, y)$ coincides with $t(x, y)$ with a fixed probability $\pi_{x y}>1 / 2$, and that errors on different choices are mutually independent; see Coombs et al. (1967).

Since an observed choice does not necessarily coincide with its underlying true choice, paired comparisons are usually replicated a number of times. Accordingly, we assume throughout that each pair $\{x, y\} \in Z \& Z$ is presented to the subject exactly $k$ times, with $k \geqslant 1$ odd; i.e. there have been $k$ replications. Common values in experiments involving one judge are $k=3$ and $k=5$. We write $x P_{j} y$ if $x$ is preferred to $y$ in precisely $k-j$ replications, where $P$ denotes the majority preference relation, i.e. $x P y$ if $x P_{j} y$ for some $j \leqslant(k-1) / 2$. Because $k$ is odd, $x$ is majority preferred to $y$, or $y$ to $x$, for every pair $\{x, y\}$ presented to the judge.

Of special interest are the stable choices, i.e. those paired comparisons in which the same choice is made in all replications. For instance, in an experiment on the seriousness of crimes, a judge generally will have no difficulty in choosing the 
most serious crime from murder and driving through a red light. In other words, if $x$ is murder, and $y$ is driving through a red light, then $\pi_{x y}$ is very high and $x P_{0} y$ very likely.

\section{The construction of a transitive order}

Our method has been motivated by the following assumptions; comments are given below.

Assumption 4.1. True preferences are acyclic.

Assumption 4.2a. The more intense an observed preference is, the more likely it is to coincide with its corresponding true preference.

Assumption 4.2b. Stable preferences have priority over unstable preferences.

Assumption 4.1 requires no explanation. As regards Assumption 4.2a, under the probabilistic assumption described above it is plausible to interpret the intensity of a choice to be monotone with $\pi_{x y}$ and to consider a majority preference as more intense the larger $k-j$ (the number of replications showing the majority choice in $\{x, y\})$ is. For Assumption 4.2b, think of choosing the most serious crime from murder and driving through a red light. Here, there will be no (or exceptionally small) random errors in preferences, and observed preferences will be stable. Obviously, stability of preferences does not preclude random errors. Instability of preferences, however, does ensure random errors. Hence, stable preferences should have special priority over unstable ones. Indeed, under the probabilistic assumption described above, for $\pi_{x y}=0.6,0.7,0.8$, the likelihood ratio $\pi_{x y}^{k} /\left(1-\pi_{x y}\right)^{k}$ is, respectively, 3.4, 12.7, 64.0 for $k=3$, and $7.6,69.2$, 1024.0 for $k=5$. These values support the priority of stable preferences but, of course, there is no certainty that a stable majority preference coincides with its corresponding true choice.

As a consequence of Assumption 4.1, transitive closure will be applied after every step in the algorithm. As a consequence of Assumptions 4.1 and 4.2a, a preference will be overruled by the transitive closure of more intense preferences. As a consequence of Assumption 4.2b, stable preferences will never be reversed. If stable preferences contain cycles, we reckon with a genuine intransitivity. Rather than imposing transitivity, we then recommend reconsidering Assumption 4.1 or the data. The possibility of revealing genuine intransitivities is in our opinion an advantage of binary choices over the direct ranking of the objects. 
Practise shows that the direct ranking of objects is problematic, probably because this is more remote from everyday experience than binary choices.

If stable preferences are acyclic, the method is applied. It constructs a sequence of transitive digraphs

$$
D_{0}, \ldots, D_{i}, \ldots, D_{f}, \quad f \leqslant n(n-1) / 2,
$$

with an increasing number of arcs. The final result $D_{f}$ gives the solution, i.e. a transitive relation. Under completeness (all pairs of objects have been presented to the judge) $D_{f}$ will be a tournament; thus the constructed transitive relation has preserved completeness. For $D_{0}$, the starting digraph, we take the digraph corresponding to the transitive closure of the stable preferences.

For the remainder of the procedure, see the flowchart in Fig. 1. A majority preference $x P_{j} y$ is called incorporated in $D_{i}$ if either the arc $x y$ or the arc $y x$ is present in $D_{i}$. In the latter case ( $y x$ present) we say a preference reversal has occurred. Now suppose that some $D_{i}$ has been constructed. If all majority preferences have been incorporated in $D_{i}$, the method stops. Therefore, suppose some majority preference has not yet been incorporated. The method then proceeds by selecting one of the most intense of these majority preferences, say $x P_{j} y$, inserting the arc $x y$, and extending by transitive closure. The particular choice from these most intense majority preferences is motivated by considerations of efficiency. Details are as follows.

Take the minimal $j$ such that a majority preference $x P_{j} y$ has not yet been incorporated. (Note that this integer $j$ must be increased in comparison with the similar $j^{*}$ used in the construction of $D_{i-1}$ only if all majority preferences $P_{j^{*}}$ have been incorporated in $D_{i}$.) Determine the score vector $s\left(D_{i}\right)=$ $(\operatorname{od}(1), \ldots, \operatorname{od}(l), \ldots, \operatorname{od}(n))$, where nodes $x_{1}, \ldots, x_{n}$ have been reordered such that $\operatorname{od}(1) \geqslant \cdots \geqslant \operatorname{od}(n)$. For the moment, the ranking of nodes with equal outdegrees is arbitrary (this arbitrariness will be dealt with below). An ordering based on outdegrees is also used in Rubinstein (1980), Roberts (1990), and Delver et al. (1991). We will often refer to $x_{l}$, the node corresponding to od $(l)$, as $l$. Note that $x_{l}$ in $s\left(D_{i}\right)$ need not refer to the same node as $x_{l}$ in $s\left(D_{i+1}\right)$, because the ordering of outdegrees depends on $D_{i}$. Pairs of nodes are written with the highest outdegree first, and are subsequently ranked in a reversed lexicographic way, i.e. as $(1,2),(1,3), \ldots,(1, n),(2,3), \ldots,(2, n), \ldots,(n-1, n)$. In this order we choose the first pair $(x, y)$ in which the $P_{j}$-relation has not yet been incorporated in $D_{i}$. Note that the search for the pair $x y$ can often be done faster; for instance, if $\operatorname{id}(l)+\operatorname{od}(l)=n-1$, node $l$ can be ignored because, then, arcs with all other nodes are already present in $D_{i}$. If $x P_{j} y$, the arc $x y$ is inserted; if $y P_{j} x$, the arc $y x$ is inserted. Note that inserting $x y$ (or, similarly, $y x$ ), cannot create a cycle: the remainder of that cycle would constitute a path from $y$ to $x$ in $D_{i}$, and, hence, by transitivity of $D_{i}$, an arc $y x$. This cannot be. Hence transitive closure can be applied, resulting in the digraph $D_{i+1}$. 


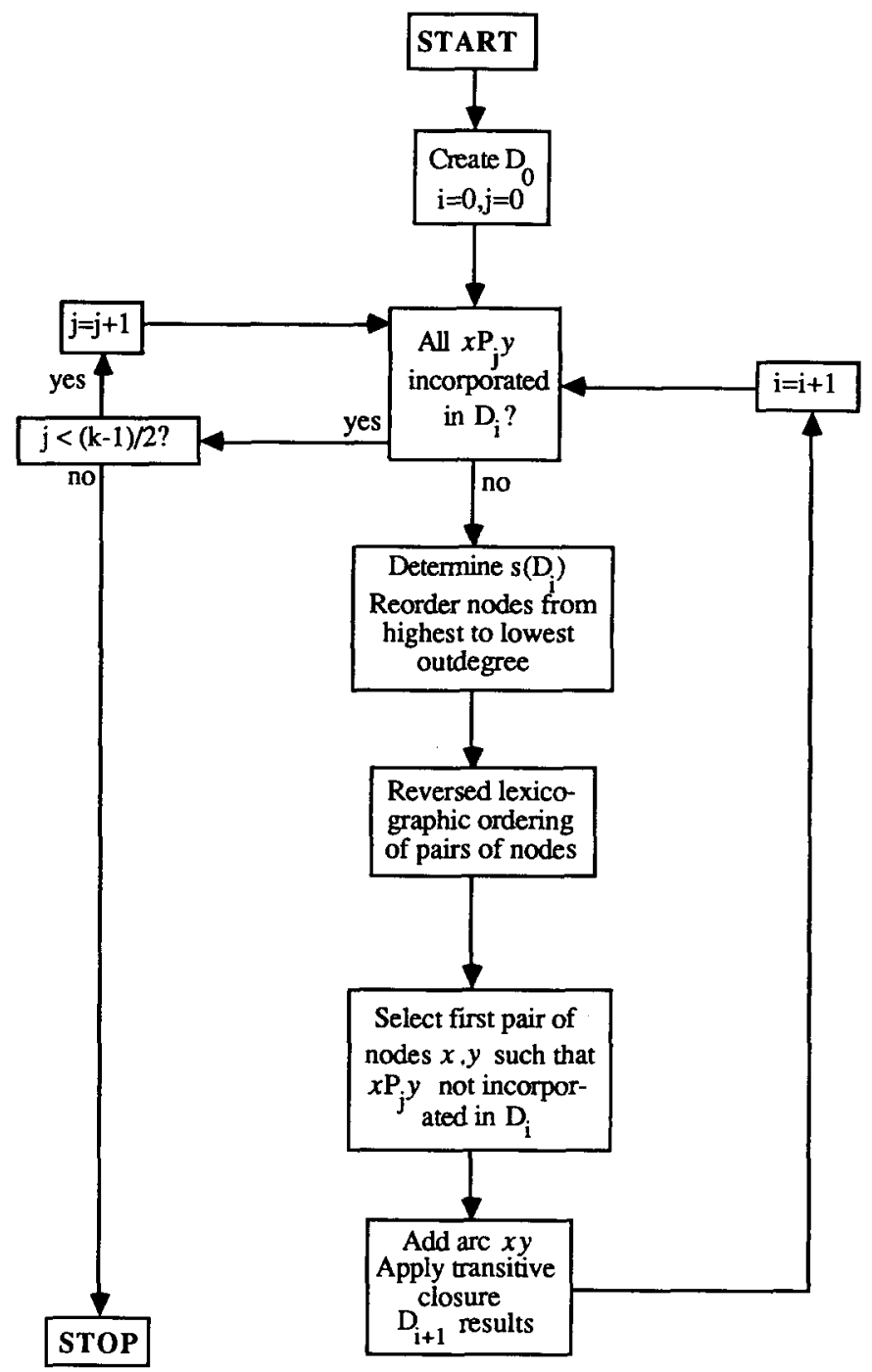

Fig. 1. Flowchart of the method.

After a finite number of steps the method stops, and all majority preferences have been incorporated. Note that the selected pair of nodes is always ordered in accordance with the majority preference. Reversals only occur through transitive closure, in agreement with the consequence of Assumptions 4.1 and $4.2 \mathrm{a}$ as described below those assumptions. As an example, if $x P_{0} y, y P_{0} z$, and $z P_{1} x$, then $x \rightarrow z$ will result.

Let us now deal with the arbitrariness of the ordering of outdegrees as 
mentioned above. Suppose some nodes have an identical outdegree. Then these are ordered by increasing indegrees. Of course, in a computer program the score vector $s\left(D_{i}\right)$ can immediately be constructed in this way. Now there will be an unambiguous ordering of nodes in most cases, and $x$ and $y$ are selected. If there still does not exist an unambiguous ordering, the method may select one pair at random. Then possibly the solution is not unique, though often the solution will be not be affected by the particular choice of a pair. This completes the description of the method.

Uniqueness can be further increased by a modification which has been used in the simulations. The price for this greater uniqueness is that the computation time is nonpolynomial. Roberts (1990) emphasizes that uniqueness is a problem for many combinatorial optimization procedures. Let us now describe the modification. All pairs of nodes that can be selected are considered. One of those pairs is selected and the solution is determined. Next another pair is selected, and again the solution is determined. This is done for all possible pairs. Finally, the solution with the minimal number of majority preference reversals is chosen. If there are two or more such solutions, then no unique solution has been obtained. However, such occurrences are rare (see Section 7). Note that if $\operatorname{od}(1)>\operatorname{od}(2)>\operatorname{od}(3)=$ $\operatorname{od}(4)=\operatorname{od}(5)$, then the ordering of $x_{3}, x_{4}$, and $x_{5}$ need not be determined immediately because the first selection may concern nodes 1 and 2 . If by this selection 12 or 21 is incorporated, then this will usually affect the ordering of $x_{3}, x_{4}$, and $x_{5}$.

The procedure is greedy, i.e. it makes the best possible choice at each single step. This does not guarantee the finding of optimal solutions, but will usually lead to solutions close to optimal solutions. The solution is locally optimal in the sense of Assumption 4.2, i.e. reversals of arcs ('pairwise interchanges') will not improve the fulfillment of Assumption 4.2.

Finally, in order to reduce calculations, it is convenient to partition the digraph representing the majority preferences (arc $x y$ if and only if majority preference $x P y$ ) into 'strong components'. A digraph is strong if it contains a walk through all nodes with the same beginning and endnode. A subdigraph of a digraph $D$ is a digraph whose nodes and arcs are nodes and arcs of $D$. A strong component $D_{1}$ of a digraph $D$ is a maximal strong subdigraph, i.e. there is no strong subdigraph $D_{2}\left(\neq D_{1}\right)$ of $D$ that contains all nodes and arcs of $D_{1}$. Obviously, cycles in majority preferences only occur within strong components of the digraph that represents the majority preference relation. Between strong components all majority preferences have the same direction, and the strong components can be ordered correspondingly. Hence it suffices to apply the method to each single strong component; next the solutions of all these separate strong components are embedded in one overall transitive solution in an obvious way. 


\section{Polynomial computation time}

As mentioned in the introduction, David (1988) qualifies the computation of all nearest adjoining orders as a 'formidable challenge'. The procedures of Remage and Thompson (1966) and Phillips (1969) require exponential computation time (see Phillips, 1969, p. 100). For many procedures it is not known whether or not they are NP (Even, 1979). Roberts (1990) expresses a strong suspicion that the 'linear ordering problem' is NP. Our method will now be shown to require only polynomial computation time. If, as in our method, the number of operations to arrive at a solution varies, the order of the polynomial is to be determined for the case in which the number of operations is maximal. Below, for each step in the method as shown in Fig. 1, the highest number of operations required to move to the next step is determined.

In the appendix it is shown that adding one arc to a transitive digraph, followed by transitive closure, requires a calculation time proportional to $n^{2} / 4$, i.e. is an $n^{2}$-algorithm. Thus, the construction of $D_{0}$ (add one arc after the other, and apply transitive closure each time) is an $n^{4}$-algorithm. Let us now consider the extension of $D_{i}$ to $D_{i+1}$, with symbols as in Section 4. Finding $j$, establishing $s\left(D_{i}\right)$, and selecting $x$ and $y$ are all $n^{2}$-algorithms. Adding the arc between $x$ and $y$ plus transitive closure is, according to the appendix, an $n^{2}$-algorithm. Hence the extension of $D_{i}$ to $D_{i+1}$ is an $n^{2}$-algorithm. Such an extension of a digraph occurs at most $n(n-1) / 2$ times (see formula (4.1)). Hence the method is an $n^{4}$ algorithm, i.e. it is polynomial. Indeed, our method has been programmed in FORTRAN with a fixed number of $n$-dependent (nested) loops, with no transitions between loops. It is well known that this ensures a polynomial computation time (Garey and Johnson, 1979).

\section{Examples}

Example 6.1. Fig. 2(a) shows observed preferences which form the 4-cycle of majority preferences ${ }^{2} P x P y P z P w$; Slater's $i$ is 1 (reverse $z w$ ). The relations $P_{0}$ and $P_{1}$ are given in Fig. 2(b). $D_{0}$ can be created on the basis of $P_{0}$, as shown in Fig. 2(c). Note that transitive closure does not result in any more arcs. Now, $s\left(D_{0}\right)=(2,0,0,0)$, in which the first 0 is the outdegree corresponding to node $w$, because of its lowest indegree ( $\mathrm{id}(w)=0)$. The ordering of pairs to be selected is $(x, w),(x, y),(x, z),(w, y),(w, z),(y, z)$, where it is noted that the ordering of $(x, y),(x, z)$, and $(w, y),(w, z)$ is arbitrary. It is easily verified that an arc between $x$ and $w$ is not yet present, so $(w, x)$ is selected. The preference $w P_{1} x$ (see Fig. $2(\mathrm{~b}))$ is incorporated as the arc $w x$. On the basis of transitive closure, $w y$ and $w z$ are added, resulting in digraph $D_{1}$ (see Fig. 2(c)). Now the only $P_{1}$-preference not yet incorporated is $y P_{1} z$; this is incorporated subsequently. Note that the 


\begin{tabular}{c|c|c|c|c|}
\multicolumn{2}{c}{$w$} & \multicolumn{1}{c}{$x$} & $y$ & $z$ \\
\cline { 2 - 5 }$w$ & - & 2 & 2 & 1 \\
\hline$x$ & 1 & - & 3 & 3 \\
\hline$y$ & 1 & 0 & - & 2 \\
\hline$z$ & 2 & 0 & 1 & - \\
\hline
\end{tabular}

(a)

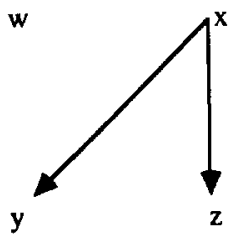

(b)
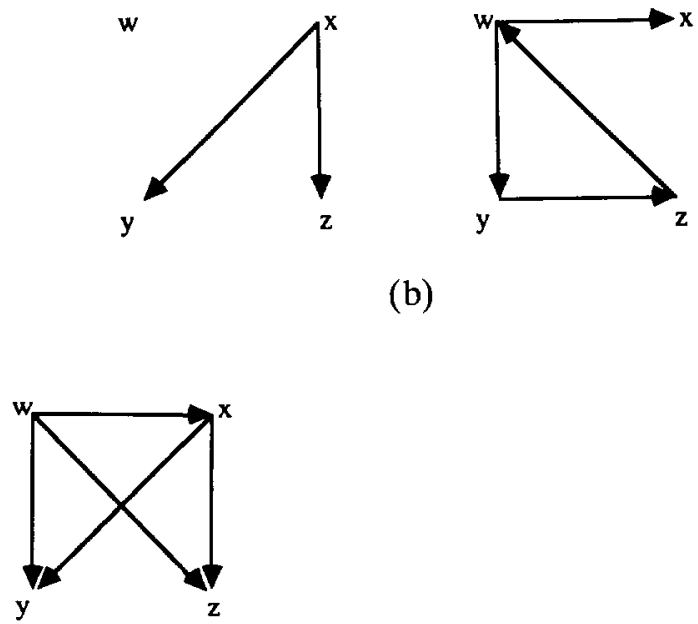

(c)

Fig. 2. (a) Preference matrix of Example 6.1. The values of the cells represent the number of times the row object is preferred over the column object in three replications. The solution of our method is wxyz. This is a nearest adjoining order. (b) Preference relations $P_{0}$ (left) and $P_{1}$ (right). (c) Digraphs $D_{0}$ (left) and $D_{1}$ (right).

ambiguity in the ordering of $y$ and $z$ in $s\left(D_{0}\right)$ does not affect the uniqueness of the solution. A nearest adjoining order has been obtained. One majority preference $z P w$ has been reversed into the arc $w z$.

Example 6.2. Suppose we have observed all preferences between every pair of four objects $w, x, y$ and $z$ in three replications. The resulting preference matrix is given in Fig. 3. Note that the matrix can be represented by a 4-cycle $w P x P y P z P w$. Slater's $i$ is 1 , and there is only one nearest adjoining order, namely the one that can be created by reversing the majority preference $z P w$, hence constituting the linear order $w x y z$. However, applied to majority preferences, Slater's procedure does not reckon with the intensities of the preferences, i.e. all arcs are taken as equal intensity. From a psychological standpoint this is debatable. After all, the

\begin{tabular}{|c|c|c|c|c|}
\multicolumn{2}{c}{$w$} & \multicolumn{1}{c}{$x$} & $y$ & $z$ \\
\cline { 2 - 5 }$w$ & - & 2 & 2 & 0 \\
\hline$x$ & 1 & - & 3 & 3 \\
\hline$y$ & 1 & 0 & - & 3 \\
\hline$z$ & 3 & 0 & 0 & - \\
\hline
\end{tabular}

Fig. 3. Preference matrix of Example 6.2. The values of the cells represent the number of times the row object is preferred over the column object in three replications. The solution of our method is $x y z w$, involving two (unstable) preference reversals. The nearest adjoining order is $w x y z$, involving one (stable) preference reversal; Slater's $i=1$. 
judge has three times preferred $z$ over $w$, i.e. the majority preference $z P w$ is highly intense. If our method is applied, then it is easily seen that, because there is a path $x y z w$ in $D_{0}$, the linear order $x y z w$ emerges from transitive closure. This implies that we reverse $w P y$ and $w P x$. In both $\{w, x\}$ and $\{w, y\}$ the observed preferences are not the same in all replications. Hence the majority preferences in these pairs show more uncertainty than does the majority preference in $\{w, z\}$. Our method thus reckons with intensities of preferences, and is appropriate if priority is given to the higher reliability of more intense preferences. In this case the solution is a transitive tournament, but not a nearest adjoining order.

Let us consider the above preferences according to the model of Tversky (1969). Suppose the objects have two dimensions, which are ordered a priori with respect to their importance, and a judge uses the following decision rule (see Fig. 4). If the difference between two objects on the first dimension is strictly greater than $2 \varepsilon$, choose the object with the higher value. If no decision based on dimension I can be made, choose the object with the higher value on dimension II, provided that the difference is strictly greater than $\varepsilon$. If, according to this decision rule, no preference between objects can be stated, then choose at random. This rule yields the stable preferences $z P_{0} w$ (based on dimension $\mathrm{I}$ ), $x P_{0} z, x P_{0} y$ and $y P_{0} z$ (based on dimension II), while in the pairs $\{w, x\}$ and $\{w, y\}$ a random choice is made. This decision rule could result in the preference matrix given in Fig. 3. In this case it would be undesirable to change the stable preferences.

Example 6.3. The matrix in Fig. 5 results in majority preferences between 12 objects enclosed in one single strong component. This strong component comprises the cycle $l P k P j P i P g P f P c P a P b P e P d P h P l$. Slater's $i$ is $4 . P_{0}$ contains no cycles, hence $D_{0}$ can be created and our method can be applied. In- and outdegrees of $D_{0}$ are given in Fig. 6. It is left to the reader to verify that, after application of our method, the linear order lkjihgefdcab results. This order is a nearest adjoining order (i.e. contains four preference reversals). The small digits in the cells of Fig. 5 indicate which edges have been directed in the same 'run' (i.e. resulting from a selection of nodes and subsequent transitive closure).

This example has been taken from a medical study of ours in which patients are

\begin{tabular}{ccc}
\multicolumn{4}{c}{ Dimensions } \\
& $I$ & II \\
$w$ & $\varepsilon$ & $4 \varepsilon$ \\
$x$ & $2 \varepsilon$ & $5 \varepsilon$ \\
$y$ & $3 \varepsilon$ & $3 \varepsilon$ \\
$z$ & $4 \varepsilon$ & $\varepsilon$
\end{tabular}

Fig. 4. The description of the objects in Fig. 3, based on three dimensions; dimensions I and II are important for the decision rule. 


\begin{tabular}{|c|c|c|c|c|c|c|c|c|c|c|c|c|c|}
\hline & $a$ & $b$ & $c$ & $d$ & $e$ & $f$ & $g$ & $h$ & $i$ & $j$ & $k$ & $l$ & $\mathbf{o}_{\mathbf{i}}$ \\
\hline$a$ & - & 210 & 0 & 0 & 0 & 0 & 0 & 0 & 0 & 0 & 0 & 0 & 1 \\
\hline$b$ & 1 & - & 1 & 1 & 2 & 0 & 1 & 0 & 1 & 0 & 0 & 0 & 1 \\
\hline$c$ & 3 & 29 & - & 1 & 1 & 0 & 0 & 0 & 1 & 0 & 0 & 0 & 2 \\
\hline$d$ & 3 & 28 & 27 & - & 0 & 0 & 0 & 2 & 0 & 0 & 1 & 0 & 4 \\
\hline$e$ & 3 & $1^{6}$ & 26 & 3 & - & 26 & 0 & 1 & 0 & 1 & 1 & 0 & 4 \\
\hline$f$ & 3 & 3 & 3 & 3 & 1 & - & 0 & 1 & 2 & 0 & 0 & 0 & 5 \\
\hline$g$ & 3 & 21 & 3 & 3 & 3 & 3 & - & 1 & 0 & 0 & 0 & 0 & 6 \\
\hline$h$ & 3 & 3 & 3 & $1^{5}$ & 25 & 25 & 25 & - & 0 & 1 & 0 & 2 & 7 \\
\hline$i$ & 3 & $\begin{array}{ll}2 & 1\end{array}$ & 21 & 3 & 3 & $1^{1}$ & 3 & 3 & - & 1 & 1 & 0 & 7 \\
\hline$j$ & 3 & 3 & 3 & 3 & 21 & 3 & 3 & 24 & $\begin{array}{ll}2 & 4\end{array}$ & - & 0 & 0 & 9 \\
\hline$k$ & 3 & 3 & 3 & 21 & 21 & 3 & 3 & 3 & $\begin{array}{ll}2 & 3\end{array}$ & 3 &. & 1 & 10 \\
\hline$l$ & 3 & 3 & 3 & 3 & 3 & 3 & 3 & $\begin{array}{ll}1 & 1\end{array}$ & 3 & 3 & 22 & - & 10 \\
\hline & 10 & 10 & 9 & 7 & 7 & 6 & 5 & 4 & 4 & 2 & 1 & 1 & \\
\hline
\end{tabular}

Fig. 5. (Example 6.3: A preference matrix with 12 nodes, each cell represents the number of times the object in the row is preferred to the object in the column). A cell marked with ${ }^{x}$, where $x$ is a number between 1 and 10, means that the edge representing this cell was directed in digraph $D_{x}$, either by adding an arc or due to transitive closure. The arc is directed from row object to column object. The preference reversals are underlined.

to choose between two treatments for laryngeal cancer, namely radiotherapy and surgery. To help patients in choosing, they are given paired comparisons of possible outcomes of treatments in which one treatment has a higher life expectancy and a worse speech quality than the other. This finally leads to a preference for one of the two treatments (Maas and Stalpers, 1992). Our procedure can be used to establish a transitive order if, after three replications, intransitivities are present.

So far, paired comparisons have been presented to nine patients. One of them stopped cooperation after one replication. From the remaining eight patients, three were lexicographic and two more were completely transitive. Hence three patients were intransitive. Judging by a measure developed by Maas (1993), one of them was 'too' intransitive. That is, the percentage of circular triads was higher than five. From one of the remaining two intransitive patients, the above example has been taken. The other patient had seven objects comprised in a single strong component. The solution based on our method requires four preference reversals,

\begin{tabular}{ccccccccccccc} 
nodes & $a$ & $b$ & $c$ & $d$ & $e$ & $f$ & $g$ & $h$ & $i$ & $j$ & $k$ & $l$ \\
\hline $\operatorname{od}(x)$ & 0 & 0 & 1 & 1 & 2 & 4 & 6 & 3 & 8 & 7 & 9 & 10 \\
$\operatorname{id}(x)$ & 10 & 7 & 7 & 7 & 5 & 5 & 4 & 3 & 1 & 2 & 0 & 0
\end{tabular}

Fig. 6. Example 6.3: Out- and indegrees in $D_{0}$, i.e. after transitive closure of the stable preferences. 
yielding a nearest adjoining order. (For the corresponding preference matrix of this patient, see Maas and Stalpers, 1992.)

Example 6.4. Suppose there are 101 objects $\left\{x_{1}, \ldots, x_{100}, w\right\}$, and there are $k=11$ repetitions. The preferences $x_{i}>x_{i+j}$ are stable for all $i>0, j>0$. For $i>1, x_{i} P_{5} w$, i.e. $x_{i}$ is chosen over $w$ six times, suggesting that $w$ should be the least preferred object. However, suppose that $w P_{4} x_{1}, w$ is preferred over $x_{1}$ seven times; this suggests that $w$ should be the most preferred object. Our algorithm first establishes the transitive ordering $x_{1}>\cdots>x_{100}$, and next incorporates $w>x_{1}$. By transitive closure, the final ordering is $w>x_{1}>\cdots>x_{100}$.

Discussion. The example clearly illustrates that our method assigns priority to intenser preferences, irrespective of the number of less-intense preferences that must be reversed subsequently. In this example, the outcome of our method seems questionable, and the final ordering $x_{1}>\cdots>x_{100}>w$ seems more plausible.

A more sophisticated variation of our method could be developed to provide a more plausible ordering. Then at each stage a new preference to be incorporated would not necessarily be the most intense preference, but would rather be determined in some sense by the number of less-intense preferences that might be reversed by incorporating the preference and transitive closure. However, such a method would be much more cumbersome, and would require subjective choices concerning tradeoffs between numbers of preference reversals and intensities of preferences. This would necessarily introduce some subjective interpretation of the importance of reversals and intensities. We have developed our method to be tractable and transparent, at the price of giving nonoptimal results in some extreme cases. Note that the above example is not natural, and that similar phenomena will occur only rarely.

Our method can be applied to social choice, where there are $k$ individuals and each individual expresses a preference, leading again to $k$ observed preferences for each pair of objects. The ordering to be constructed is now interpreted as a group preference relation. A feature of our method is that it ensures the unanimity principle, i.e. if all persons agree on a preference between two objects, then so will the group preference. Our method is, however, not strategy-proof, i.e. it may be in a subject's interest to represent his or her preference incorrectly. This is illustrated in the following example.

Example 6.5 (strategic behavior in a social choice). Suppose, as in Example 6.4, that there are 101 objects $\left\{x_{1}, \ldots, x_{100}, w\right\}$. There are $k=11$ persons, and the preferences $x_{i}>x_{i+j}$ are unanimous (i.e. stable) for all $i>0, j>0$. The first person has a transitive preference relation $x_{1}>w>x_{2}>\cdots>x_{100}$. Suppose that $w P_{5} x_{1}, x_{i} P_{5} w$ for $i=2, \ldots, 99$, and $x_{100} P_{4} w$. Our algorithm first establishes 
$x_{1}>x_{2}>\cdots>x_{100}$, then incorporates $x_{100}>w$; by transitive closure, $x_{1}>x_{2}>\cdots>$ $x_{100}>w$ finally results. If the first person were to know this, then he or she might prefer revealing the preferences $w>x_{1}>x_{2}>\cdots>x_{100}$. In that case, again our algorithm first establishes $x_{1}>x_{2}>\cdots>x_{100}$; in the next step, either $w P_{4} x_{1}$ or $x_{100} P_{4} w$ is to be incorporated, and because $x_{1}$ has a higher outdegree than $x_{100}$, the preference $w>x_{1}$ is incorporated. By transitive closure, $w>x_{1}>x_{2}>\cdots>$ $x_{100}$ results, which person 1 may well prefer over the ordering $x_{1}>x_{2}>\cdots>$ $x_{100}>w$. It is then to person 1's advantage to misrepresent his/her true preferences.

\section{Monte Carlo study}

We assume that the true latent preferences are represented by a real function $f$, i.e. $x$ is preferred to $y$ if and only if $f(x)>f(y)$. Manifest choices, denoted by $>$, are probabilistic in accordance with Luce's (1959) model, i.e.

$$
\pi(x>y)=\frac{f(x)}{f(x)+f(y)},
$$

where $\pi(x>y)$ is the probability of choosing $x$ over $y$ in a single choice. Scale values $f(x), f(y)$ are chosen such that for all $x, y$ with consecutive scale values the probabilities $\pi(x>y)$ are equal. Hence, for objects $x, y, z, \ldots, v, w$ (with decreasing scale values), the following equations need to be solved:

$$
\frac{f(x)}{(f(x)+f(y))}=\frac{f(y)}{(f(y)+f(z))}=\cdots=\frac{f(v)}{f(v)+f(w)} .
$$

Without loss of generality, $f(x)$, being the highest scale value, can be fixed at 1.0. The lowest scale value, $f(w)$, is fixed at 0.001 (not at 0 , because $\pi(x>w)=1.0$ is not allowed in Luce's model). Now, (7.1) becomes

$$
\frac{1}{(1+f(y))}=\frac{f(y)}{f(y)+f(z)}=\cdots=\frac{f(v)}{f(v)+.001} .
$$

With $n$ objects, the solution of $(7.2)$ is $f(y)=[f(w)]^{1 /(n-1)}$, and $f(z)=$ $[f(y)]^{2}, \ldots, f(v)=[f(y)]^{n-2}$.

The results of the Monte Carlo study are given in Table 1. It is obvious that, especially for low values of $n$, our method almost always produces a nearest adjoining order. This is partially due to the fact that probabilities are rather high. The proportion of nearest adjoining orders gradually decreases with increasing $n$, and is around 0.50 when $n=15$. Proportions do not vary much over 3 and 5 
Table 1

Results of the Monte Carlo study with Luce's choice model

\begin{tabular}{rlllll}
\hline$n$ & $k$ & Proportion & Interval $^{\mathrm{a}}$ & Cyclic & Not unique \\
\hline 5 & 3 & 0.971 & $0.85-1.0$ & 0 & 0 \\
7 & 3 & 1.0 & $0.93-1.0$ & 0 & 0 \\
7 & 5 & 1.0 & $0.90-1.0$ & 0 & 0 \\
9 & 3 & 0.893 & $0.82-0.94$ & 0.016 & 0 \\
9 & 5 & 0.938 & $0.86-0.97$ & 0.004 & 0 \\
11 & 3 & 0.818 & $0.76-0.87$ & 0.015 & 0.005 \\
11 & 5 & 0.811 & $0.73-0.87$ & 0 & 0 \\
13 & 3 & 0.606 & $0.51-0.69$ & 0.033 & 0.008 \\
13 & 5 & 0.614 & $0.52-0.70$ & 0.008 & 0 \\
15 & 3 & 0.501 & $0.40-0.60$ & 0.101 & 0 \\
15 & 5 & 0.462 & $0.36-0.56$ & 0.010 & 0 \\
\hline
\end{tabular}

Notes: $n=$ number of objects, $k=$ number of replications, proportion = proportion nearest adjoining orders resulting from solution; Interval ${ }^{a}=5 \%$ confidence interval for proportions of previous column; Cyclic $=$ proportion of digraphs showing cyclic stable preferences; and Not unique $=$ the proportion of matrices in which no unique solution could be determined.

${ }^{\mathrm{a}}$ For this interval the usual formula $p \pm 1.96 \mathrm{~V} p(p-1) / n$ is used.

replications. The number of cyclic $P_{0}$ is low; the number of digraphs with nonunique solutions with an equal number of preference reversals is very low.

\section{Discussion}

It has been shown that the method presented gives a solution that often is unique, and often coincides with a nearest adjoining order, especially for $n<10$. The Monte Carlo study supports the method. It will be of use in practice if researchers and/or judges adhere to a formal decision, or want to prepare data, in the context of a prespecified model that assumes transitivity. In contrast to the algorithms for finding all nearest adjoining orders of Remage and Thompson (1966) and Phillips (1969), our method is polynomial and therefore suitable for large numbers of objects. In practice the method is straightforward and can be done by hand, even with 15 objects. It has not been our aim to arrive at nearest adjoining orders because, as shown in Example 6.2, a nearest adjoining order need not be a proper solution if intensities of preferences are relevant.

As we have seen, the number of arc reversals in one empirical case (Example 6.3) coincides with Slater's $i$. For otner methods such an example, consisting of a strong component with 12 nodes, is complex We have collected more real data as in Example 6.3, and until now our method always produced a nearest adjoining order. The method fairly often gives one solution established in a few minutes without a computer.

The requirement of replications can be modified by asking respondents to directly state intensities of preferences on a, say, 3- or 5-point scale. The 
applicability of the method to social choice was described in Example 6.5 and the text above.

If high numbers of replications are involved, then the method can be modified. It can then be made more tractable by collapsing the $P_{j}$-relations. For example, let $P_{0}^{*}:=P_{0}$, let $P_{1}^{*}$ be the union of $P_{1}, \ldots, P_{k_{1}}, P_{2}^{*}$ the union of $P_{k_{1}+1}, \ldots, P_{k_{2}}$, etc. Another possibility is to enlarge the class of preferences to be treated as the stable preferences in our method. For instance, if the relative choice frequency for a particular choice significantly exceeds randomness (Coombs et al., 1967), then this may be treated as a stable choice. The remaining choices are then treated as the unstable preferences in Section 4.

All examples and applications given so far concerned tournaments. We give two examples of incomplete preference structures to which our method can be applied.

Example A. If we collect dominances of similarities between pairs $\{x, y\}$, with $x$ fixed, $y \neq x$, then these could be interpreted as meaning that if $\{x, y\}>\{x, z\}$, then $x$ and $y$ are judged to be more similar than $x$ and $z$. With $n$ nodes, there are $M=\left(\begin{array}{c}n-1 \\ 2\end{array}\right)$ such paired comparisons with fixed $x$. These $M$ comparisons form a tournament $T_{x}$. We similarly obtain $T_{y}, T_{z}, \ldots$, yielding $n$ tournaments altogether. Such dominances of similarities are typically derived from a 'conditional proximity matrix', or result directly from 'conjoint paired comparisons' (Bezembinder, 1991). Such data may easily show cycles, in particular between tournaments. For instance, it is possible that $\{y, x\} \rightarrow\{x, z\}$ within $T_{x}$ and $\{x, z\} \rightarrow\{z, y\}$ within $T_{z}$, but $\{z, y\} \rightarrow\{y, x\}$ within $T_{y}$. As for distinct $x, y, z, w$, no arc between $\{x, y\}$ and $\{z, w\}$ is given in the data, methods for solving intransitivities are called for that, like our method, do not require completeness in the data.

Example B. Complete bipartite digraphs. These are denoted by $D\left(V_{1}, V_{2}\right)$, in which $V_{1}$ and $V_{2}$ are disjoint sets of objects such that there is an arc connecting every node of $V_{1}$ with every node of $V_{2}$, while there are no other arcs. An example is Guttman's (1944) scalogram with $n$ subjects and $m$ items, which can be represented by such a structure. Here our method can also be applied. Relations between nodes of the same set should then not be inserted as a result of transitive closure.

The main motivation for developing our method has been to provide a solution that is widely applicable because:

- It is easily obtained in little (polynomial) time, can easily be programmed, and can even be calculated by hand for numbers of objects that are not too large.

- It has a transparent psychological basis because it reckons with stability/ intensity of preference, and avoids the confounding of stable preferences with the, evidently unreliable, unstable preferences. 


\section{Acknowledgements}

The research of Peter Wakker has been made possible by a fellowship of the Royal Netherlands Academy of Arts and Sciences. Two anonymous referees gave helpful comments; Example 6.4 was provided by one referee.

\section{Appendix: The maximum number of directed arcs resulting from transitive closure}

Say $D$ is a transitive digraph with $n$ nodes. An arc between $x$ and $y$ is not yet present in $D$, and the arc $x y$ is to be added. Let $A$ be the set containing $x$ and all nodes from which an arc goes to $x$. Let $B$ be the set containing $y$ and all nodes $b$ such that an arc goes from $y$ to $b . A$ and $B$ are disjoint because $y z x$ for $z \in A \cap B$ together with [not $y x$ ] would violate transitivity of $D$. Say $A$ contains $s$ nodes, $B$ contains $t$ nodes. Transitive closure will generate at most $s t-1$ new arcs, i.e. all nodes from $A$ to $B$ except $x y$. This is maximal for $s=t=n / 2$. So all together, at most $n^{2} / 4$ arcs are added.

\section{References}

I. Ali, W.D. Cook and M. Kress, On the minimum violations ranking of a tournament, Manage. Sci. 32 (1986) 660-672.

J.-P. Barthélemy, Intransitivities of preferences, lexicographic shifts and the transitive dimension of oriented graphs, Brit. J. Math. Stat. Psychol. 43 (1990) 29-37.

D.E. Bell, Regret in decision making under uncertainty, Oper. Res. 30 (1982) 961-981.

Th. Bezembinder, Circularity and consistency in paired comparisons, Brit. J. Math. Stat. Psychol. 34 (1981) 16-37.

Th. Bezembinder, Circularity in conjoint paired comparisons, in: J.-C. Falmagne and J.P. Doignon, eds, Mathematical Psychology: Current Developments (Springer, Berlin, 1991) pp. 157-180.

C.H. Coombs, Th. Bezembinder and F.M. Goode, Testing expectation theories of decision making without measuring utility or subjective probability, J. Math. Psych. 4 (1967) 72-103.

H.A. David, The Method of Paired Comparisons, 2nd edn. (Griffin, London, 1988).

R. Delver, H. Monsuur and A.J.A. Storcken, Ordering pairwise comparison structures, Theory and Decision 31 (1991) 75-94.

S. Even, Graph Algorithms (Pitman, London, 1979).

P.C. Fishburn and I.H. LaValle, A nonlinear, nontransitive and additive-probability model for decisions under uncertainty, Ann. Stat. 15 (1987) 830-844.

M.R. Garey and D.S. Johnson, Computers and Intractability: A Guide to the Theory of NPCompleteness (Freeman, San Francisco, 1979).

S.T. Goddard, Ranking tournaments and group decisionmaking, Manage. Sci. 29 (1983) 1384-1392.

L. Guttman, A basis for scaling qualitative data, Amer. Sociological Rev. 9 (1944) 139-150.

L. Hubert, Seriation using asymmetric proximity measures, Brit. J. Math. Stat. Psychol. 29 (1976) 32-52.

M.G. Kendall and B.B. Smith, On the method of paired comparisons, Biometrika 31 (1940) 324-345. 
G. Loomes and R. Sugden, Regret theory: An alternative theory of rational choice under uncertainty, Econom. J. 92 (1982) 805-824.

R.D. Luce, Individual Choice Behavior (Wiley, New York, 1959).

A. Maas, A Relativized Measure of Circularity, Math. Soc. Sci. 26 (1993) 79-91.

A. Maas and L.J.A. Stalpers, Assessing utilities by means of additive conjoint measurement: An application in medical decision analysis, Medical Decision Making 12 (1992) 288-297.

J.W. Moon, Topics on Tournaments (Holt, Rinehart and Winston, New York, 1968).

J.P.N. Phillips, A further procedure for determining Slater's $i$ and all nearest adjoining orders, Brit. J. Math. Stat. Psychol. 22 (1969) 97-101.

R. Remage, Jr. and W.A. Thompson, Jr., Maximum-likelihood paired comparison rankings, Biometrika 53 (1966) 143-149.

J.M. Roberts, Jr., Modeling hierarchy: Transitivity and the linear ordering problem, J. Math. Sociol. 16 (1990) 77-87.

A. Rubinstein, Ranking the participants in a tournament, SIAM J. Appl. Math. 38 (1980) 108-111.

J. Singh and W.A. Thompson, Jr., A treatment of ties in paired comparisons, Ann. Math. Stat. 39 (1968) 2002-2015.

P. Slater, Inconsistencies in a schedule of paired comparisons, Biometrika 48 (1961) 303-312.

A. Tversky, Intransitivity of preferences, Psychol. Rev. 76 (1969) 31-48. 